\title{
Optimized profile planar UWB monopole antenna for optimal adaptation
}

Jose Martinez-Fernandez, Jose M. Gil, and Juan Zapata

Dpto. Electromagnetismo y Teoria de Circuitos

Universidad Politecnica de Madrid

ETSI Telecomunicacion

Ciudad Universitaria S/N

28040 Madrid, Spain

Email: jmf@etc.upm.es; jmg@etc.upm.es; jzapata@etc.upm.es

\section{Introduction}

This work deals with the possibility of making a completely blind optimization of the profile of a planar monopole antenna in order to satisfy previously defined demands, in this case minimal return losses for an UWB system. Previous results in this topic were already discussed in [1]. In that work, optimization of the profile of the antenna was not completely blind due to the high computational effort needed to obtain results, which can turn to be a complete failure. Hence, to speed up the method, the global optimization was performed using a less accurate but quicker analysis and then refining the solution with a local optimization using a very accurate analysis.

Here, a pure global optimization using the same design methodology is presented. In this case, each iteration of the global optimization is performed using enough computatinal effort for the solution to be accurate. This means increased design times but also a refined solution which leads to a better antenna performance.

\section{Theory}

This design methodology makes use of a global optimization algorithm and a versatile and fast analysis technique for each optimization step. The global optimization is carried out by a Simulated Annealing algorithm [2] and for the analysis tool, the Finite Element Method (FEM) with Segmentation/Finite Element Padè via Lanczos (SFELP) [3] formulation was used. The method used in this paper is a generalization of the $2 \mathrm{D}$ revolution-symmetry-based method presented in [4].

\section{Numerical and Experimental Results}

As a result from the optimization process, an optimized profile UWB has been designed in order to obtain the minimum return losses in the $2-11 \mathrm{GHz}$ frequency band. To make the optimization, infinite ground plane computations were used in order to reduce the amount of time required in each optimization loop. Figure 1 shows the profile of the antenna in a $3-\mathrm{D}$ view surrounded by the spherical port, which is the geometry used for the analysis.

Better than $18 \mathrm{~dB}$ return losses were obtained in the optimization step. In order to validate them, a prototype was built and measured. The optimized profile was built 


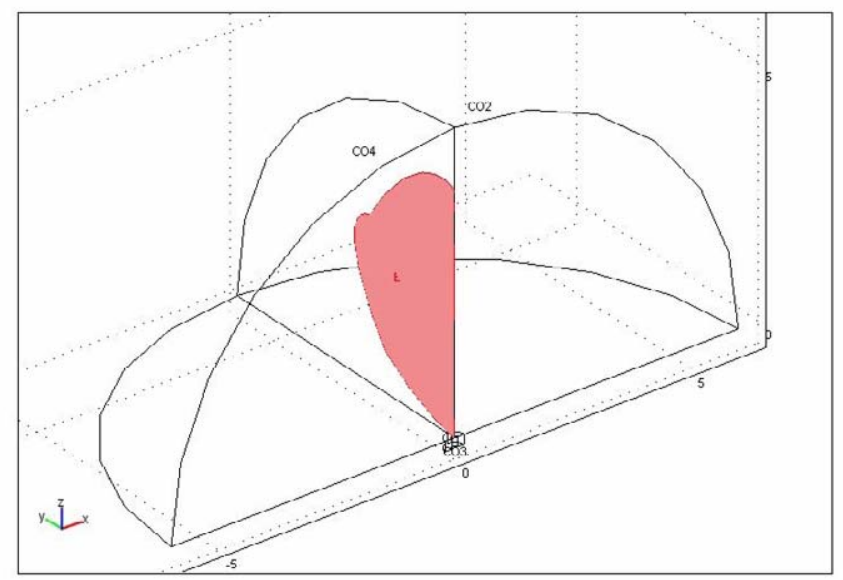

Figure 1: Antenna profile. 3-D view

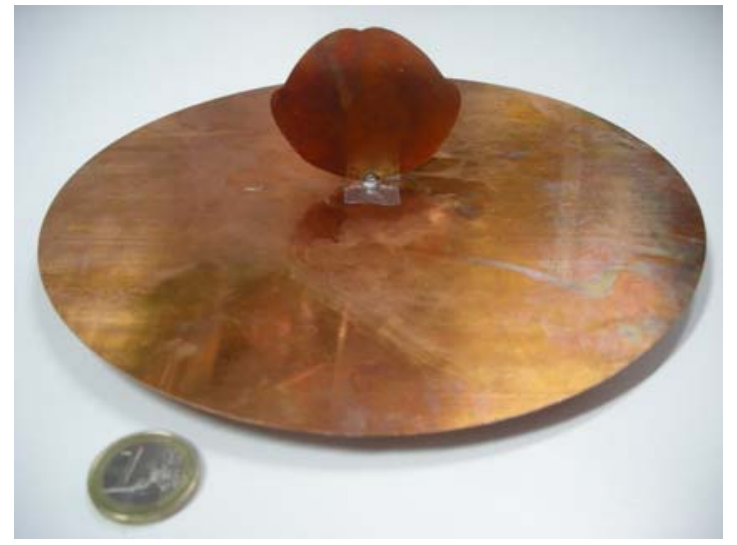

Figure 2: Photograph of the prototype compared with an euro coin.

using a copper sheet of $0.5 \mathrm{~mm}$ thickness and then attached to a SMA connector. A $10 \mathrm{~cm}$ radius ground plane was chosen due to manufacturing constraints. This means an electrical length near $\lambda$ in the lower part of the frequency band. Figure 2 shows a photograph of the built prototype including the ground plane and one euro coin for dimension comparison purposes.

Figure 3a presents return losses of the designed antenna both simulated with an infinite ground plane and measured with a $10 \mathrm{~cm}$. radius ground plane. Return losses of the antenna were measured using a HP-8510C network analyzer. Simulated results are presented in a dashed line and measurements are given in a solid line. Return losses below 18dB have been achieved in the whole frequency band. A good agreement between numerical and experimental results can be seen. Differences between both can be explained taking into account the small electrical length of the ground plane.

Results from the previous figure were presented in the $2-11 \mathrm{GHz}$ frequency band, 


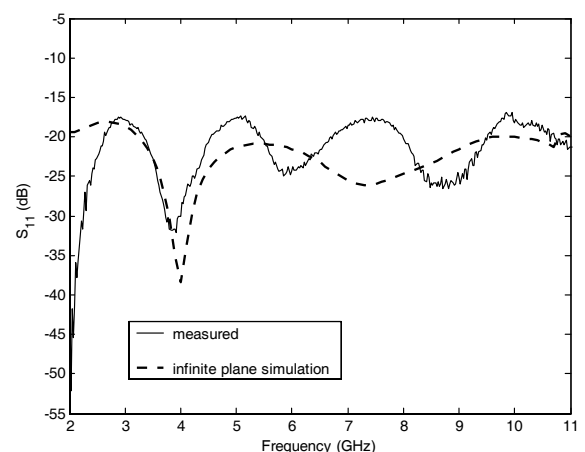

a) Infinite ground plane simulation and $10 \mathrm{~cm}$ ground plane measured return losses of the antenna.

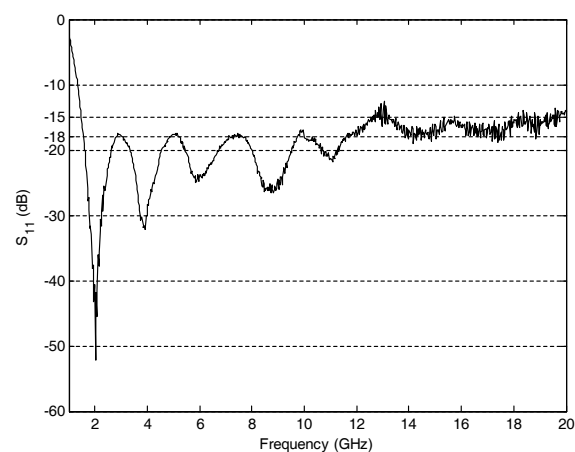

b) Extended return losses measurements in the $1-20 \mathrm{GHz}$ band

Figure 3: Return losses results

which was the optimized frequency band. These results can be extended measuring the behavior of the antenna in a broader band. Figure $3 \mathrm{~b}$ shows return losses of the antenna in the $1-20 \mathrm{GHz}$ band. Better than $15 \mathrm{~dB}$ return losses were obtained in all the band.

Figure 4 presents computed radiation patterns of the antenna in each of the principal cuts at four equally spaced frequencies. Both copolar and crosspolar components ( $\mathrm{E}_{\theta}$ and $\left.\mathrm{E}_{\phi}\right)$ are taken into account. In case of the $\phi=0^{\circ}, 90^{\circ}$ planes, the crosspolar component is negligible, so there is no plot for it. Radiation patterns are only plotted till $\theta=90^{\circ}$ because analysis has been made using an infinite ground plane.

It is important to note that there is a ripple effect that increases as frequency gets higher. This effect reflects the behavior of a standard monopole high above its resonance frequency. Regardless this ripple effect, it should be noted that the pattern remains stable in the directions of maximum radiation $\left(\theta= \pm 90^{\circ}\right)$. This is a very important result for the use of this antenna in UWB systems. In addition, these directions are also the ones with lesser levels of crosspolar radiation. Nevertheless crosspolar levels are not a common limitation in UWB designs because of the common existance of multipath enviroment.

\section{Conclusion}

An optimized profile UWB monopole antenna for use in the $2-11 \mathrm{GHz}$ band has been designed, built and measured. Better than 18dB return losses have been computed and measured in a valid prototype. Good agreement between computer analysis and measurements has been found. Radiation pattern analysis shows a monopole-like behaviour, with an increasing ripple as frequency gets higher. Fortunately, radiation patterns remain stable for the $\theta= \pm 90^{\circ}$ which is the direction of maximun radiation and therefore the direction of interest. All these characteristis make this antenna and excellent choice for UWB systems needing very low return losses, a planar profile 

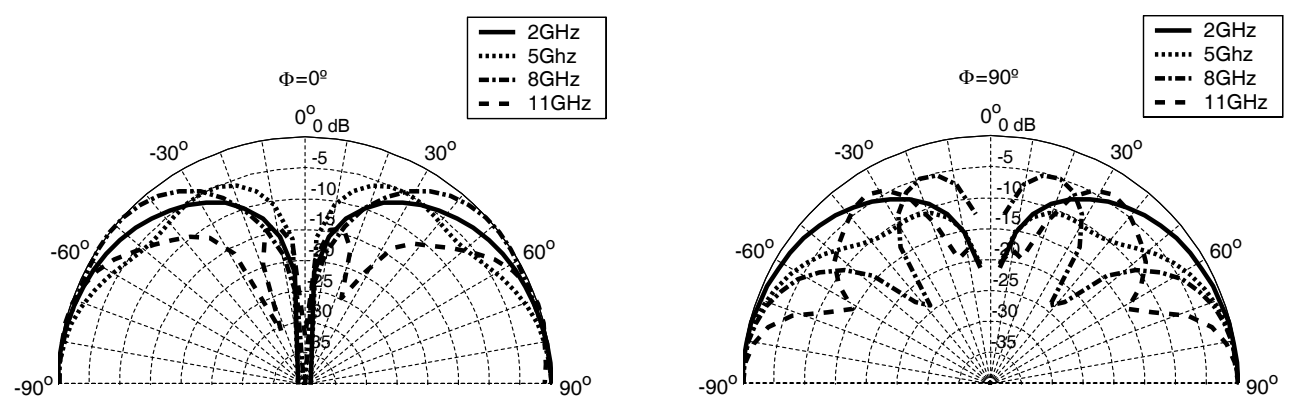

$\phi=0^{\circ}$. Copolar component $\left(E_{\theta}\right)$.

$$
\phi=90^{\circ} \text {. Copolar component }\left(E_{\theta}\right) .
$$
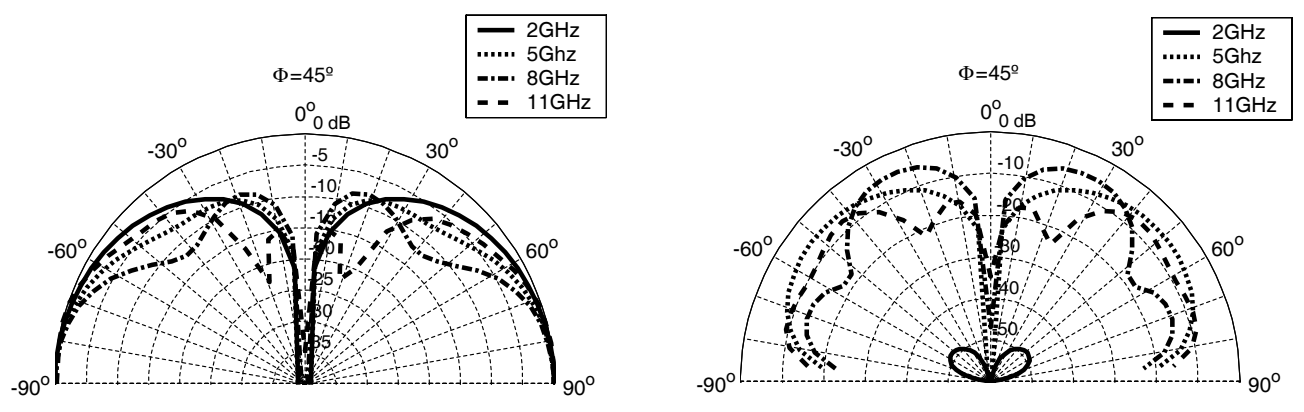

$\phi=45^{\circ}$. Copolar component $\left(E_{\theta}\right) . \quad \phi=45^{\circ}$. Crosspolar component $\left(E_{\phi}\right)$.

Figure 4: Simulated radiation patterns of the antenna using an infinite ground plane for different frequencies in each principal plane

and omnidirectional monopole like radiation patterns.

\section{References}

[1] J. Martínez-Fernández, J. M. Gil, and J. Zapata, "Optimization of the profile of a planar ultra wide band monopole antenna in order to minimize return losses," in Proc. of the 2nd European Conference on Antennas and Propagation (EuCAP), Nov. 2007.

[2] R. H. J. M. Otten and L. P. P. P. van Ginneken, The Annealing Algorithm. Boston/Dordrecht/London: Kluwer Academic Publishers, 1989, p. 201.

[3] J. Rubio, J. Arroyo, and J. Zapata, "SFELP-an efficient methodology for microwave circuit analysis," IEEE Trans. Microwave Theory Tech., vol. 49, no. 3, pp. 509-516, Mar. 2001.

[4] J. Martínez-Fernández, J. M. Gil, and J. Zapata, "Ultra wide band optimized profile monopole antenna by means of simulated annealing algorithm and the finite element method," IEEE Trans. Antennas Propagat., vol. 55, no. 2, pp. 1826-1832, June 2007. 